\title{
What can we learn from vibrational analysis calculations of defective polymer chains?
}

\author{
H.M.G. CORREIA ${ }^{\mathrm{a}}$, AND M.M. D.Ramos ${ }^{\mathrm{a}}$ \\ ${ }^{a}$ Departamento de Física, Universidade do Minho, \\ Campus de Gualtar, 4710-057 Braga, Portugal
}

\begin{abstract}
The possibility of using infrared (IR) spectroscopy to determine the concentration of inversion monomer defects in polymers depends on the knowledge of the relationship between the spectral properties and the polymer microstructure. This can easily be achieved by performing vibrational analysis.

In order to investigate the changes in IR spectra of poly(vinylidene fluoride) resulting from the presence of monomeric units in "head-to-head" and "tail-to-tail" positions, we calculated the frequencies and intensities of IR-active vibrations for individual molecules in alpha and beta form with a defect concentration up to $15 \%$ and compared them with the ones obtained for a defect-free molecule.
\end{abstract}

Keywords: PVDF; Atomistic modeling; monomer inversion; Infrared spectroscopy

\section{INTRODUCTION}

Since the discovery of the piezoelectric properties of poly(vinylidene fluoride) (PVDF) in 1969 by Kawai [1], this polymer has been under intensive investigation concerning its physical properties and applications.

PVDF exhibits three main polymorphic forms primarily differentiated by chain conformation. Besides, the addition of a certain percentage of inverted or reversed monomer units during the polymerization of vinylidene fluoride leads to the presence of constitutional defects in the PVDF chains. Therefore, an actual PVDF sample, depending on the preparation conditions, may present one or more polymorphic forms in addition to a given defect content. 
Among the spectroscopic techniques suitable to distinguish the different polymorphic forms of PVDF and the presence of polymer chain defects, assuming the form of head-to-head and tail-to-tail inversions, infrared spectroscopy is the most often used. The molecular vibration modes of $\alpha, \beta$ and $\gamma$ conformations of PVDF and the alternating copolymer of ethylene and tetrafluoroethylene (a typical model compound for $\beta$-PVDF chains with head-to-head and tailto-tail defects), calculated by Kobayashi et al. [2] in 1975 using a valence force field and a procedure called normal coordinate analysis [3], have been widely used to identify the absorption peaks in the infrared spectrum of PVDF. Different calculated normal frequencies were obtained recently using the Hartree-Fock self-consistent field method [4] and its semiempirical application called CNDO (Complet Neglect of Differential Overlap) method [5]. Therefore, the contradictory data published in the literature may be due to different calculation methods employed.

Despite all this effort, there are still unsolved problems concerning the interpretation of the infrared spectrum of PVDF. The observed spectrum cannot be analyzed by simply comparing the calculated normal frequencies of $\alpha, \beta$ and $\gamma$ defect-free chains with the observed data, since vibrational coupling can occur between the reversed monomer units and the otherwise completely head-to-tail sequence.

The aim of this work is to perform vibrational frequency calculations of alpha and beta-PVDF chains with and without monomer inversion defects to assess the influence of defect concentration and localization in the chain on the calculated infrared spectrum of PVDF.

\section{THEORETICAL METHOD}

The commercial quantum chemistry program HyperChem (Hypercube Inc., Canada) with a semi-empiricized version of the Hartree-Fock self-consistent field method, called CNDO [6], 
was used to perform both the geometry optimization and the vibration analysis of $\alpha$ - and $\beta$ PVDF chains with and without head-to-head and tail-to-tail constitutional defects.

HyperChem models the vibrations of the polymer chain as a set of $\mathrm{N}$ point masses (the nuclei of the atoms), each of them vibrating about its optimized position. The optimized positions were previously determined by solving the electronic Schrödinger equation using the CNDO method.

Using Newton's second law, a system of $3 \mathrm{~N}$ simultaneous linear differential equations is obtained, which can be transformed into the matrix from

$$
\mathbf{U L}=\mathbf{L} \Lambda
$$

where $\mathbf{U}$ is a matrix of mixed partial second derivatives of the total energy with respect to the nuclear positions (force constants), $\mathbf{L}$ and $\Lambda$ are eigenvectors and eigenvalues respectively. The matrix $\mathbf{U}$ is then diagonalized to yield normal modes of vibration and their corresponding frequencies. In the numerical evaluation of second derivatives, HyperChem uses the energies and forces evaluated quantum mechanically by the CNDO method.

A normal mode can absorb infrared (IR) light if the molecular dipole moment changes in the course of a normal vibration. In HyperChem, the following equation is used for calculating the intensity of IR-active vibrations:

$$
A_{k}=\frac{\pi N_{A} g_{k}}{3000 \ln (10) c^{2}}\left|\frac{\partial \mu}{\partial Q_{k}}\right|^{2}
$$

where $N_{A}$ is the Avogadro number, $c$ is the light velocity, $g_{k}$ is the degeneracy factor and $\partial \mu / \partial Q_{k}$ is the dipole moment derivative with respect to the normal coordinate displacement $Q_{k}$.

As noted before, whilst the CNDO method gives good optimized geometries, it tends to overestimate vibrational frequencies [7]. The advantage of this method is that such overestimate is systematic, so relative frequencies are reliable. However, we must be cautious 
in comparing calculated vibrational frequencies directly with those observed in experimental IR spectra.

Despite the errors associated with the limited basis set used for the quantum mechanical calculations, notably in vibrational frequencies, we believe it is essential to use a selfconsistent tried and tested method, where ranges of validity and accuracy are well established, to investigate the effects of different chain conformation and the presence of constitutional defects on IR spectra of PVDF. This has the particular virtue of avoiding fitting answers and the use of empirical inter-atomic potentials that are usually not well known.

\section{RESULTS AND DISCUSSION}

In order to study the effect of chain length on the IR spectrum of both alpha- and beta-PVDF, we have calculated the IR spectrum for chains with a number of monomer units ranging from 2 to 20 . Results of these calculations show three mains features. First, the number of IR-active vibrations associated with the stretching, bending, twisting, rocking and wagging of $\mathrm{CH}_{2}, \mathrm{CF}_{2}$ and $\mathrm{C}-\mathrm{C}-\mathrm{C}$ increases when chain length increases. Second, the vibration coupling between the repeat units results in spectral bands that have frequency depending on the length of the chain. Third, the intensity of the IR lines also increases with chain length.

Since the agreement between the calculated and the measured IR spectrum of PVDF improves with an increase in chain length [4], we will present here only results obtained for a chain with 20 monomers units.

IR spectroscopy has been widely used to characterize the presence of different conformers in a PVDF sample [8-13]. The IR spectrum of a mixture of conformers will exhibit bands arising from the molecular vibrations of all conformers. The assignment of an IR absorption band to a specific conformer is based on the normal vibrational analysis of defect-free chains. However, the addition of a certain percentage of inverted monomer units in the polymer chains, which is 
an unavoidable phenomenon during polymerization, can generate spectral interferences that are not well understood.

A deeper insight into the effect of head-to-head and tail-to-tail defects on the IR spectrum of PVDF can be obtained by performing vibrational-mode analysis of isolated PVDF chain with the two most common conformations, zigzag all-trans conformation ( $\beta$-form) and transgauche-trans-gauche conformation ( $\alpha$-form).

In order to obtain a reliable and consistent reference frame, we calculate first the IR spectrum of a defect-free PVDF chain. The optimized geometry obtained for $\alpha$ - and $\beta$-PVDF chains with 20 monomer units is depicted in figure 1 and their calculated IR spectra are show in figure 2. By comparing both spectra we can easily distinguish the $\alpha$-form from the $\beta$-form. The calculated $\alpha$-PVDF spectrum has several peaks in the region that extends to $340 \mathrm{~cm}^{-1}$ (region A), a peak located at $635 \mathrm{~cm}^{-1}$ (region $\mathrm{B}$ ), two bands in the region that extends from 2000 to $2500 \mathrm{~cm}^{-1}$ (region C) and no peaks in the region that extends from 4500 to $5000 \mathrm{~cm}^{-1}$ (region $\mathrm{D}$ ), whereas the $\beta$-PVDF chain has no peaks in region $\mathrm{A}$ and $\mathrm{B}$, a single band in region $\mathrm{C}$ and several peaks in region $\mathrm{D}$.

(a)

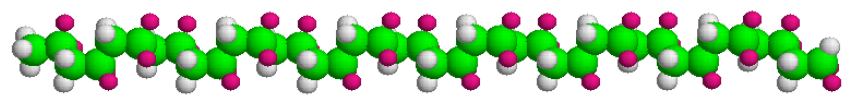

(b)

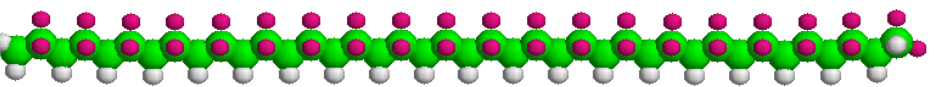

FIGURE 1. The optimized molecular geometry of a defect-free PVDF chain with 20 monomer units and the following molecular conformations: (a) $\alpha$-form; (b) $\beta$-form. 


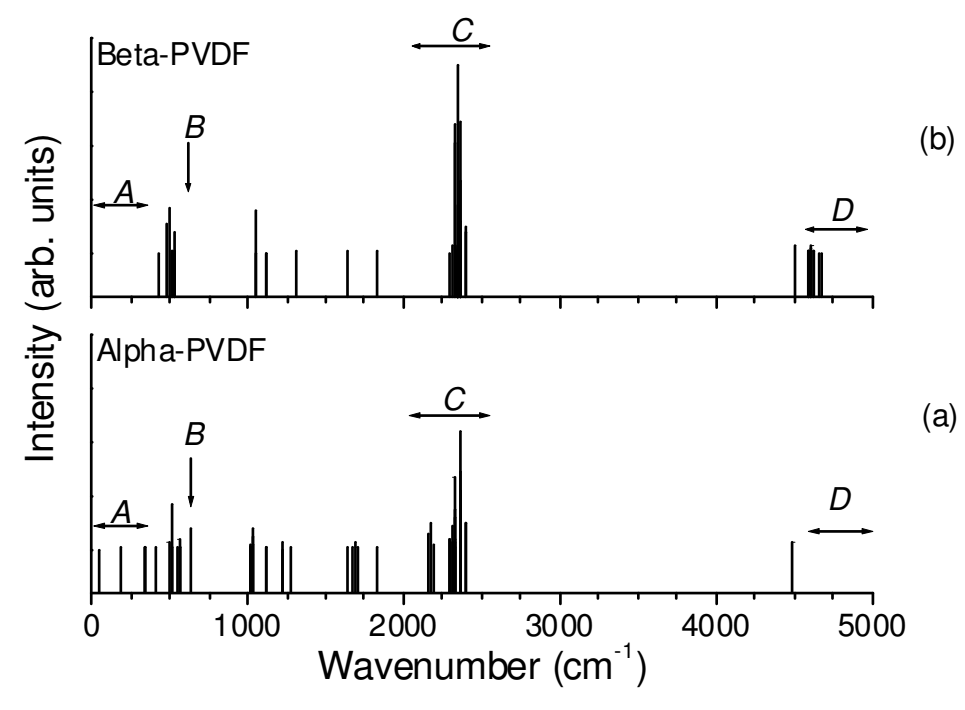

FIGURE 2. The calculated infrared vibrational spectra of defect-free PVDF chain with 20 monomer units: (a) $\alpha$-form; (b) $\beta$-form.

However, assigning the peaks in regions $\mathrm{A}$ and $\mathrm{B}$ to $\alpha$-form and the peaks in region $\mathrm{D}$ to the $\beta$-form is misleading. As can be seen in figure 3, the presence of inverted monomer units in both $\alpha$ - and $\beta$-PVDF chains can change completely the interpretation of those peaks. In fact, the peaks in region $D$ that seem to be characteristic of $\beta$-chains are also present in the calculated IR spectra of $\alpha$-chains with one or more head-to-head and tail-to-tail defect. Moreover, the peak at $635 \mathrm{~cm}^{-1}$ (B) related previously to $\alpha$-form can also be present in the spectrum of a $\beta$-chain with a single inverted monomer unit in the centre of this chain. 


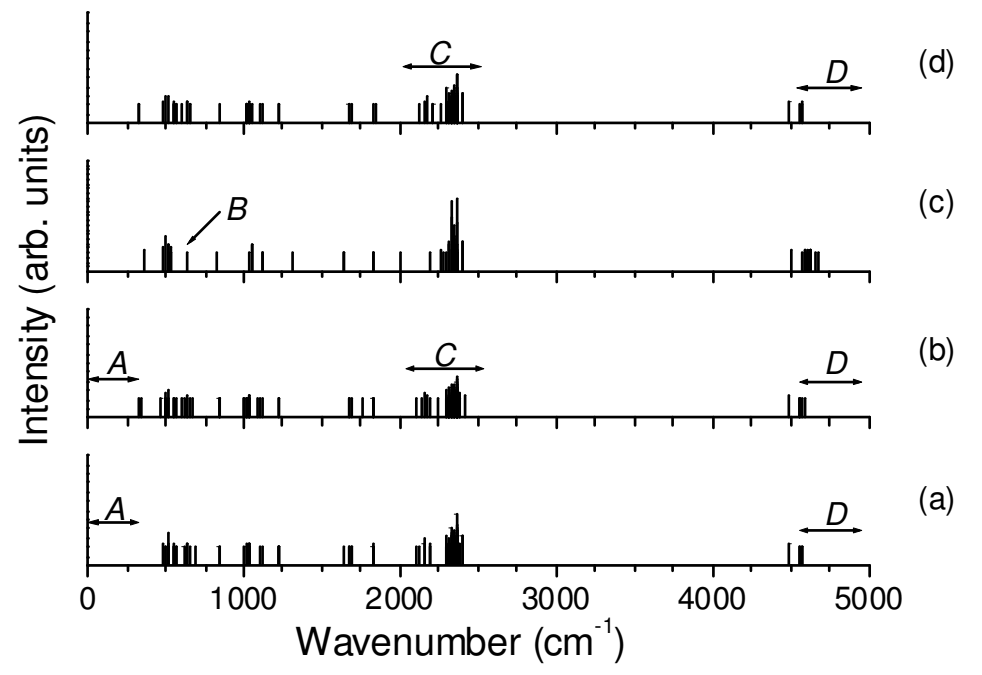

FIGURE 3. The calculated infrared vibrational spectra of the following PVDF chains: (a) $\alpha-$ chain with two isolated inverted monomer units; (b) $\alpha$-chain with three isolated inverted monomer units; (c) $\beta$-chain with a single inverted monomer unit at the centre of the chain; (d) $\alpha$-chain with a sequence of three inverted monomer units at the centre of the chain. The presence of inverted monomer units, as constitutional defects in PVDF chains is indicated unambiguously, in the calculated IR spectra of $\alpha$ - and $\beta$-PVDF chains, by the appearance of new IR-active vibrational frequencies in the region that extends from 820 to $850 \mathrm{~cm}^{-1}$ (see figure 4), corresponding to the rocking mode of $\mathrm{CH}_{2}$ at tail-to-tail linkages. In addition, the number of new peaks in this region is equal to the number of tail-to-tail defects, regardless of the type of molecular conformation. 


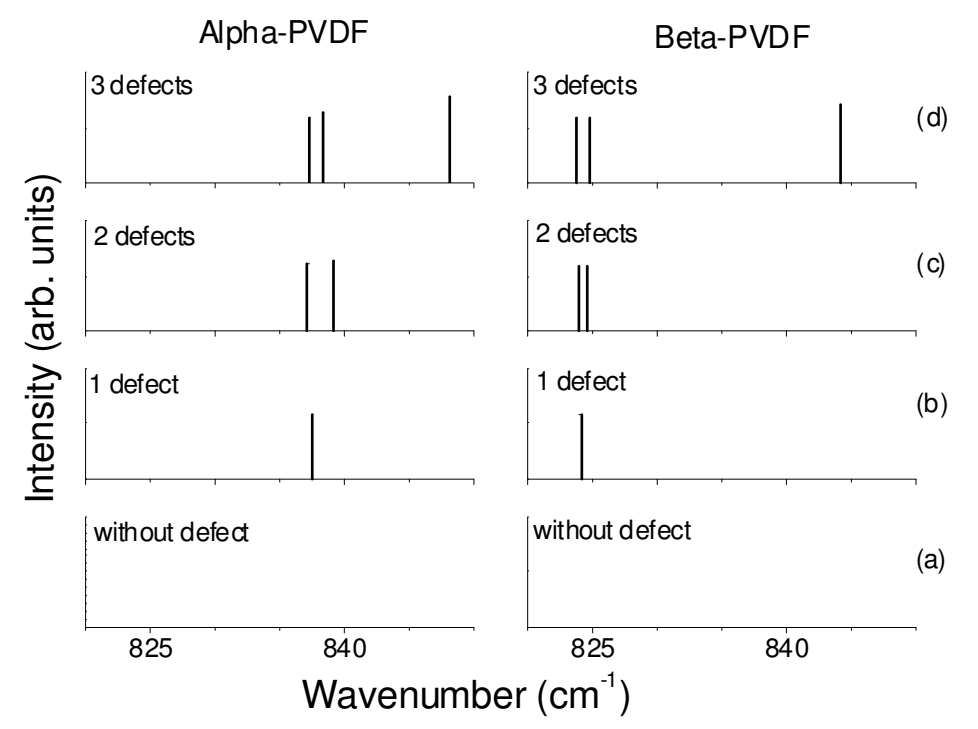

FIGURE 4. The calculated infrared vibrational spectra at the wavenumber region $820-850$ $\mathrm{cm}^{-1}$ for $\alpha$-PVDF and $\beta$-PVDF chains without inverted monomer units (a) and with isolated inverted monomer units ranging from 1 to 3 (b-d).

Figure 5 shows that the presence of constitutional defects, assuming the form of head-to-head and tail-to-tail defects, also gives rise to the splitting of the defect-free peak located between 1820 and $1860 \mathrm{~cm}^{-1}$ into two or more distinct peaks. The width between the spectral multiplets provides information for the discrimination of the distance between head-to-head and tail-to-tail defects. These results suggest that a head-to-head defect will be followed immediately by a tail-to-tail concatenation if the width between adjacent multiplets is smaller that $15 \mathrm{~cm}^{-1}$. Moreover, the peak at $1313 \mathrm{~cm}^{-1}$, which seems to be characteristic of the $\beta$ chains, is not slitting by the presence of the constitutional defects. 


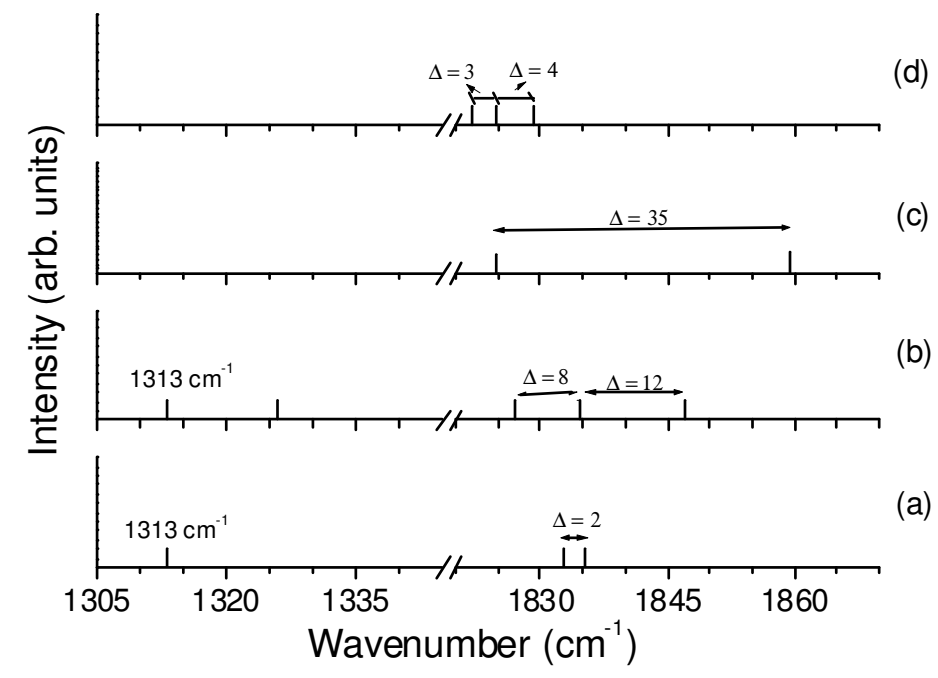

FIGURE 5. The calculated infrared vibrational spectra at the wavenumber region $1305-1345$ $\mathrm{cm}^{-1}$ and $1820-1870 \mathrm{~cm}^{-1}$ for the following PVDF chains: (a) $\beta$-chain with an single inverted monomer unit at the centre of the chain; (b) $\beta$-chain with three isolated inverted monomer units; (c) $\alpha$-chain with a sequence of three inverted monomer units at the chain end; (d) $\alpha$ chain with two isolated inverted monomer units.

Further comparison between the calculated IR spectra of $\alpha$ - and $\beta$-PVDF chains with and without constitutional defects allows one to identify other peaks that are associated with defect localization along the chains.

Our calculations clearly indicate that polymer chain defects, due to head-to-head and tail-totail linkages, are responsible for the appearance of new frequencies in IR spectra as well as the shifting, splitting and vanishing of absorption frequencies. As a result the interpretation of the IR spectrum of a given PVDF sample is not straightforward.

\section{CONCLUSIONS}

We have studied the effects of constitutional defects on IR spectra of PVDF chains with alpha and beta conformations. Our results suggest that the presence of such defects gives rise to the 
appearance of new IR peaks and shifting, splitting and vanishing of peaks already existing in defect-free chains. Therefore, one needs to be cautious in the interpretation of the IR spectrum of given PVDF samples since the assignment of an absorption peak to the calculated vibrational frequency of a given phase is not straightforward. We believe that the splitting of IR peaks observed experimentally might be related to the presence of polymer chain defects rather than the presence of a different phase.

Whilst we should not regard the vibrational frequencies calculated using the CNDO method as especially accurate, the results presented here yield much guidance of use in understanding experiments. Self-consistent calculations, such as those reported here, are a useful tool for understanding the effects of polymer chain defects on IR spectra as well as for providing data which is important for the interpretation of the experimental results.

\section{ACKNOWLEDGEMENT}

This work was supported by the Portuguese Foundation for Science and Technology (FCT) and the European Community Fund FEDER under the projects number POCTI/CTM/41574/2001 and CONC-REEQ/443/2001. One of us (H.M.G.C.) is also indebted to FCT for financial support under PhD grant no. SFRH/BD/11231/2002.

\section{REFERENCES}

[1] Kawai, H.: Piezoelectricity of Poly (Vinylidene Fluoride). Japanese Journal of Applied Physics 8 (7), 975-976 (1969).

[2] Kobayashi, M., Tashiro, K., and Tadokoro, H.: Molecular Vibrations of 3 Crystal Forms of Poly(Vinylidene Fluoride). Macromolecules 8 (2), 158-171 (1975).

[3] Wilson, E.R., Decius, J.C., and Cross, P.C.: Molecular Vibrations, New York: McGraw Hill; 1955. 
[4] Li, J.C., Wang, C.L., Zhong, W.L., Zhang, P.L., Wang, Q.H., and Webb, J.F.: Vibrational mode analysis of beta-phase poly(vinylidene fluoride). Applied Physics Letters 81 (12), 2223-2225 (2002).

[5] Correia, H.M.G. and Ramos, M.M.D.: Quantum modelling of poly(vinylidene fluoride). Computational Materials Science 33 (1-3), 224-229 (2005).

[6] Pople, J.A. and Beveridge, D.L.: Approximate Molecular Orbital Theory, New York: McGraw-Hill; 1970.

[7] Wallace, D.S., Electron-lattice coupling in conjugated polymers, in D. Phil. Thesis. 1989, University of Oxford.

[8] Bormashenko, Y., Pogreb, R., Stanevsky, O., and Bormashenko, E.: Vibrational spectrum of PVDF and its interpretation. Polymer Testing 23 (7), 791-796 (2004).

[9] Salimi, A. and Yousefi, A.A.: FTIR studies of beta-phase crystal formation in stretched PVDF films. Polymer Testing 22 (6), 699-704 (2003).

[10] Boccaccio, T., Bottino, A., Capannelli, G., and Piaggio, P.: Characterization of PVDF membranes by vibrational spectroscopy. Journal of Membrane Science 210 (2), 315-329 (2002).

[11] Virk, H.S., Chandi, P.S., and Srivastava, A.K.: Physical and chemical changes induced by $70 \mathrm{MeV}$ carbon ions in polyvinylidene difluoride (PVDF) polymer. Nuclear Instruments \& Methods in Physics Research Section B-Beam Interactions with Materials and Atoms 183 (3-4), 329-336 (2001).

[12] Lanceros-Mendez, S., Mano, J.F., Costa, A.M., and Schmidt, V.H.: FTIR and DSC studies of mechanically deformed beta-PVDF films. Journal of Macromolecular SciencePhysics B40 (3-4), 517-527 (2001). 
[13] Hilczer, B. and Kulek, J.: The effect of dielectric heterogeneity on the pyroelectric response of PVDF. IEEE Transactions on Dielectrics and Electrical Insulation 5 (1), 45-50 (1998). 